\title{
Reflets
}

Revue d'intervention sociale et communautaire

\section{Une formation en service social à distance en français : le modèle de l'Université Laurentienne}

\section{Marie-Luce Garceau et Gabriela Marcoci}

Volume 21, numéro 2, automne 2015

URI : https://id.erudit.org/iderudit/1035438ar

DOI : https://doi.org/10.7202/1035438ar

Aller au sommaire du numéro

Éditeur(s)

Reflets, Revue d'intervention sociale et communautaire

ISSN

1203-4576 (imprimé)

1712-8498 (numérique)

Découvrir la revue

Citer cet article

Garceau, M.-L. \& Marcoci, G. (2015). Une formation en service social à distance en français : le modèle de l'Université Laurentienne. Reflets, 21(2), 196-204.

https://doi.org/10.7202/1035438ar d'utilisation que vous pouvez consulter en ligne. 


\title{
Une formation en service social à distance en français : le modèle de l’Université Laurentienne
}

\author{
Marie-Luce Garceau \\ Professeure, École de service social, Université Laurentienne \\ Gabriela Marcoci \\ Professeure, École de service social, Université Laurentienne
}

Le présent article porte sur le programme d'enseignement à distance offert à l'Université Laurentienne dans le cadre du baccalauréat en service social (BSS). Ce programme s'adresse à une population francophone vivant en situation minoritaire et dispersée géographiquement. Nous en présentons un bref historique, de même que son objectif, ses points forts et ses limites.

\section{Bref historique du programme}

Au début des années 1990,l'École de service social offre quelques cours à distance, mais c'est de 2002 à 2005 que le programme de BSS à distance prend réellement son envol. Les cours obligatoires et quelques cours optionnels, développés pour la majorité en format " correspondance ", permettaient en effet de couvrir l'ensemble du programme. Concurremment, le corps professoral envisageait la mise sur pied d'un programme de formation offert entièrement en ligne, et ce, afin d'exploiter la technologie émergente et d'étendre le programme à une clientèle francophone vivant en contexte minoritaire et géographiquement éloignée des milieux universitaires. 
Le travail et le financement consacrés au développement de cours en ligne ont permis au corps professoral d'obtenir un soutien pédagogique solide. Depuis, les cours par correspondance du BSS ont été révisés, de nouveaux s'y sont ajoutés, et ils sont maintenant offerts en ligne à l'aide de la plateforme d'apprentissage Desire to Learn (D2L). Ce format de livraison des cours permet de joindre les étudiantes et étudiants francophones, disséminés sur un vaste territoire et affectés par l'éloignement géographique, tout en leur permettant de préserver leur identité linguistique et culturelle.

En 2011 et 2012, l’École de service social a procédé à une évaluation de ce programme auprès des étudiantes et étudiants actifs de même qu'auprès des diplômées et diplômés. À travers cette étude, nous souhaitions que le corps étudiant puisse décrire lui-même le programme de BSS offert à distance, ses raisons d'entreprendre des études à distance, son niveau de satisfaction quant aux divers aspects du programme (cours obligatoires, cours optionnels, stages pratiques) et, finalement, les améliorations à lui être apportées. Bref, il s'agissait de mesurer l'efficacité du programme de BSS offert à distance et en français à l'Université Laurentienne, et de voir s'il répond adéquatement aux besoins des populations rurales, urbaines et cosmopolites du Nord ontarien ou d'ailleurs.

\section{Contexte de l'étude}

La décentralisation est un desideratum de la formation universitaire du XXI ${ }^{e}$ siècle. À cet effet, la plupart des universités canadiennes offraient dans les années 1990 des cours hors campus. Une quarantaine d'entre elles offraient des cours à distance, en accord avec cette nouvelle "forme d'autodidaxie assistée qui permet à l'apprenant adulte d'accéder à des ressources médiatisées de savoirs, sans l'intervention classique d'un enseignant, mais avec le soutien d'un réseau de ressources d'encadrement " (Henry et Kaye, dans Dalceggio, 1990, p. 13). La création de programmes d'enseignement à distance au Canada se justifie, entre autres, par l'étendue du territoire à desservir, par la faible densité de la population, par la nécessité de préserver l'identité linguistique 
et culturelle de certaines populations, notamment francophones, et par le nombre limité de professeurs (Power, et collab., 1994).

\section{Résultats}

L'évaluation du programme a été réalisée à l'aide d'un sondage à questions ouvertes et fermées (administré par Survey Monkey) mené auprès de 107 étudiantes et étudiants actifs et de 40 diplômées et diplômés. Le taux de réponse a été de $60 \%$. Les variables générales de l'analyse ont été : les avantages du programme; les approches pédagogiques; les ressources; les limites; les améliorations à apporter au programme.

Les principaux thèmes ayant émergé de l'analyse du corpus sont les suivants :

\section{La mission :}

Le programme répond bien à sa mission en offrant une approche généraliste de la pratique en service social basé sur un mélange harmonieux de cours obligatoires, de cours optionnels et de stages pratiques. Le choix d'un tel programme permet aux étudiantes et aux étudiants d'augmenter l'éventail d'interventions visant directement des personnes, des familles et des groupes ou indirectement des communautés ou la société en général.

«C'est un programme extrêmement complet et professionnel. Il renferme des connaissances qui suscitent l'éveil de notre conscience face aux problématiques sociales actuelles et en lien avec l'évolution de ces dernières. ${ }^{1}$

"Il s'agit d'un programme ayant pour but de former des étudiantes et des étudiants dans le domaine social afin de leur permettre d'intervenir auprès de différentes clientèles en besoin. L'intervention peut être en clinique, communautaire, scolaire ou en agences, dépendamment de la clientèle ciblée.» 
L'alternance entre les cours et les stages pratiques a été améliorée au fil du temps pour s'assurer que les étudiantes et étudiants acquièrent les connaissances, les aptitudes pratiques et conceptuelles et un engagement professionnel propres à toute personne titulaire d'un diplôme professionnel de premier cycle.

L'organisation des stages du BSS offert à distance est effectuée par la coordonnatrice des stages de l'École de service social qui travaille en étroite collaboration avec les étudiantes et les étudiants, les milieux de stage, les consultantes académiques et les superviseures ou superviseurs des stages, afin d'en assurer le succès. Les étudiantes et les étudiants explorent les possibilités de stages dans leur milieu d'appartenance et ils en discutent avec la coordonnatrice.

"J'ai dû faire les démarches moi-même concernant mon milieu de stage. Ça m'a permis de m'intégrer et de faire des contacts avant le stage.»

\section{La qualité :}

Le corps étudiant considère qu'il est très exigeant de faire des études à distance, et que la charge de travail peut être lourde.

«Très intéressant, apprentissage approfondi, mais un besoin d'avoir beaucoup de discipline et de ne pas croire que parce que le cours est à distance qu'il y aura une certaine lacune ou un certain manque dans les apprentissages. »

"Le BSS à distance demande beaucoup plus de lectures que sur le campus et demande peut-être un effort supplémentaire, mais il en ressort une excellente formation en travail social.»

De plus, le programme est apprécié pour sa flexibilité, la disponibilité et l'engagement du corps professoral et du personnel du soutien, de même que pour la qualité de la majorité des cours.

«Les professeurs tiennent compte des situations des étudiantes et des étudiants, et sont très disponibles et engagés envers les élèves et leur réussite.» 
La variété et la qualité des stages offerts, incluant les milieux et la qualité de la supervision, sont également appréciées.

« Pour avoir été en stage en même temps que de futures travailleuses sociales, étudiantes à une autre université, je me suis sentie à leur hauteur au niveau des connaissances et habilités, même plus. En plus, nous apprenons à développer notre sens de l'organisation, d'entraide et de discipline personnelle."

\section{La pertinence :}

À la lumière de l'expérience éducative vécue, le programme de BSS à distance a un impact positif sur la carrière des étudiantes et des étudiants, sur la promotion professionnelle ou sur l'insertion en milieu du travail. Pour certaines et certains qui sont déjà actifs sur le marché du travail, cette qualification additionnelle a eu un impact positif soit sur leur emploi actuel, soit sur l'obtention d'un emploi en service social.

\section{L'accessibilité :}

Le corps étudiant souligne le fait que le programme soit offert à distance et qu'il le soit à temps plein ou à temps partiel en facilite l'accessibilité. Malgré un certain isolement, le programme offre la possibilité de rencontres.

«Je dirais que le programme est très bien organisé, que les professeurs sont disponibles et prêts à répondre à nos questions, et que le fait d'utiliser les chatrooms, les vidéos et le courriel nous fait oublier que nous n'allons pas en classe."

«J'ai connu des personnes très intéressantes et qui ont la même passion que moi, celle de vouloir faire un changement dans le monde."

«La petite taille du programme : on finit par développer des relations avec les autres étudiants.» 
" Grâce à l'université qui offre ce programme à distance je peux continuer mes études malgré mon manque de mobilité! Cela me sauve littéralement la vie en me laissant "construire" mon futur! »

Par ailleurs, le corps étudiant indique que le BSS leur offre la possibilité d'étudier en français, même s'ils habitent loin d'un centre universitaire.

«C'est le seul BSS offert entièrement à distance, entièrement francophone."

\section{Le choix d'étudier à distance :}

Pour les étudiantes et étudiants, comme pour les diplômées et diplômés, le choix d'étudier dans notre programme est orienté par la possibilité qu'offre le BSS de faire des études à distance afin de concilier ces dernières, l'emploi et la famille, ou encore parce qu'elles ou ils ne vivent pas à proximité d'un centre universitaire.

«Un programme adapté à la réalité des mamans au travail, accessible.»

«En ligne, nous avons le privilège de rester auprès de nos familles, d'économiser le temps et d'argent ayant gérer le petit budget ensemble en famille. Mais aussi, nous avons le temps d'assister des gens ayant besoin de notre service. "

Le fait que le BSS soit offert en français est aussi invoqué comme raison de leur choix de faire des études en service social. Le fait que le BSS à distance est reconnu par l'Association canadienne de formation en travail social (ACFTS) ainsi que par les ordres professionnels des diverses provinces canadiennes joue un rôle très important dans la décision d'opter pour ce programme.

\section{Aspects à améliorer :}

Tout programme a besoin d'être révisé régulièrement afin d'être amélioré. Notre programme, comme n'importe quel autre, n'est pas parfait. En ce sens, les étudiantes et étudiants ont offert 
plusieurs suggestions afin de l'améliorer et celles-ci sont connues et partagées par les membres du corps professoral du BSS à distance et par le Centre d'éducation permanente (Envision) qui gère la livraison du programme.

Ainsi, il est important de réviser régulièrement les cours afin de les mettre à jour, tout en s'assurant que certains parmi eux ne sont pas plus chargés que d'autres. Selon le corps étudiant, les échéanciers concernant les travaux ne sont pas toujours réalistes, malgré les obligations qu'impose l'université. Par ailleurs, la livraison du matériel des cours accuse parfois des retards, la disponibilité de certains professeurs est parfois déficitaire. Plusieurs mentionnent que la quantité de lectures et la charge de travail sont élevées.

«Le montant de lectures à faire, c'est trop. Dans certains cours le nombre de travaux à faire n'était pas réaliste pour le temps alloué considérant que la majorité des étudiants doivent travailler hors de la maison.»

Par ailleurs et malgré l'implication continue du corps professoral, des étudiantes ou étudiants mentionnent avoir besoin de plus d'interactions avec le corps professoral.

Dans le cadre du BSS offert à distance, les étudiantes ou étudiants doivent se présenter sur le campus dans le cadre du cours SESO2315 FL12 La relation d'aide. Pour la majeure partie du corps étudiant, il s'agit d'une occasion unique, d'une durée de deux jours et demi, de rencontrer l'ensemble de leurs collègues, les professeures ou professeurs de ce cours et l'ensemble des corps professoral et de soutien. De plus, l'objectif de cette rencontre est de permettre aux étudiantes et aux étudiants l'intégration du contenu du cours, l'expérience et la mise en pratique ainsi que d'offrir l'opportunité d'évaluer l'acquisition de leurs habiletés. Comme plusieurs vivent à de grandes distances du campus, les frais peuvent être élevés.

«Ne pas obliger les étudiants à se déplacer pour un cours sur le campus. Nous faisons le choix 
d'étudier à distance pour la diminution des coûts et la disponibilité. Le fait de devoir se rendre sur le campus quand tu habites l'extérieur est un énorme problème."

Bref, pour améliorer le programme, il faut revoir de façon satisfaisante les cours actuels de formation à distance, assurer la diversification des stages et des cours optionnels, rendre plus faciles les visites sur campus des étudiantes et étudiants et assurer l'appui nécessaire à contrer le décrochage.

\section{Conclusion}

De notre courte analyse du programme de BSS à distance nous déduisons que sa mise en place favorise un apprentissage actif et interactif et qu'il représente un modèle pédagogique efficace qui répond aux besoins actuels en matière de formation pratique en service social.

"L'École de service social forme des travailleuses sociales qualifiées. Les connaissances et compétences du corps professoral font que les diplômés ont les connaissances et habiletés pratiques, nécessaires pour promouvoir le développement humain et la lutte contre les inégalités sociales qui sont dans la société.»

Plus particulièrement, il répond aux besoins de formation en service social pour la population francophone dispersée sur le territoire canadien.

"C'est un programme accessible et flexible afin qu'il puisse s'adapter aux situations de vie changeante de l'étudiante ou étudiant. C'est un programme qui fait cheminer l'étudiante ou l'étudiant de façon progressive à travers les théories, les fondements et la pratique du travail social. Le programme nous permet 
de perfectionner plusieurs aspects du travail social, mais garde une portion pour l'étude des pratiques sociales auprès des Premières Nations.»

Pourtant, le programme a besoin d'être révisé régulièrement. Cela demande des ressources administratives et professorales qui ne sont pas toujours présentes. Finalement, il exige une coopération étroite entre le Centre d'éducation permanente (Envision) et l'École de service social, afin d'assurer le maintien du niveau de qualité le plus élevé possible.

\section{Note}

Toutes les citations sont tirées des réponses aux questions ouvertes présentées dans le sondage.

\section{Bibliographie}

DALCEGGIO, Pierre (1990). La formation à distance, Montréal, Université de Montréal, 103 p.

ÉCOLE DE SERVICE SOCIAL (2012). Rapport d'évaluation du programme à distance en vue d'obtention de l'agrément, Sudbury, Université Laurentienne, École de service social, Université Laurentienne, $30 \mathrm{p}$.

POWER, Michaël, et collab. (1994). L'encadrement des étudiants en situation d'apprentissage à distance à l'Université du Québec à Rimouski, Rimouski, Département des sciences de l'Éducation, 148 p. 